\title{
Quantum Dynamics Study of the Isotopic Effect on Capture Reactions: $\mathrm{HD}, \mathrm{D}_{2}+\mathrm{CH}_{3}$
}

\author{
Dunyou Wang* \\ Eloret, NASA Ames Research Center, \\ Mail Stor T27B-1, Moffett Field, CA 94035-1000
}

(Dated: August 7, 2002)

\begin{abstract}
Time-dependent wave-packet-propagation calculations are reported for the isotopic reactions, $\mathrm{HD}+\mathrm{CH}_{3}$ and $\mathrm{D}_{2}+\mathrm{CH}_{3}$, in six degrees of freedom and for zero total angular momentum. Initial state selected reaction probabilities for different initial rotational-vibrational states are presented in this study. This study shows that excitations of the $H D\left(D_{2}\right)$ enhances the reactivities; whereas the excitations of the $\mathrm{CH}_{3}$ umbrella mode have the opposite effects. This is consistent with the reaction of $\mathrm{H}_{2}+\mathrm{CH}_{3}$. The comparison of these three isotopic reactions also shows the isotopic effects in the initial-state-selected reaction probabilites. The cumulative reaction probabilities (CRP) are obtained by summing over initial-state-selected reaction probabilities. The energy-shift approximation to account for the contribution of degrees of freedom missing in the six dimensionality calculation is employed to obta $\mathrm{n}$ approximate full-dimensional CRPs. The rate constant comparison shows $\mathrm{H}_{2}+\mathrm{CH}_{3}$ reaction has the biggest reactivity, then $\mathrm{HD}+\mathrm{CH}_{3}$, and $\mathrm{D}_{2}+\mathrm{CH}_{3}$ has the smallest.
\end{abstract}

*Electronic address: dywang@nas . nasa.gov 


\section{INTRODUCTION}

Due to the importance of the reaction in methane combustion and pyrolysis[1], $H+$ $\mathrm{CH}_{4} \rightarrow \mathrm{H}_{2}+\mathrm{CH}_{3}$, and it: reverse reaction, $\mathrm{H}_{2}+\mathrm{CH}_{3} \rightarrow \mathrm{H}+\mathrm{CH}_{4}$ have been studied experimentally[2-11] and th soretically[13-18] since the 1960s. In addition, the reverse reaction also plays an importan role in the modeling of the chemistry of hydrocarbons in the atmospheres of giant planets[12]. Besides a number of quantum dynamics calculations [1926] in reduced dimensionality and a full-dimensional transition state flux method quantum calculation on the forward reaction $\mathrm{H}+\mathrm{CH}_{4}$, recently we reported a $6 \mathrm{D}$ time-dependent quantum study of the reverse reaction $\mathrm{H}_{2}+\mathrm{CH}_{3} \rightarrow \mathrm{H}+\mathrm{CH}_{4}[27]$.

In this reduced dimensional calculation, $\mathrm{CH}_{3}$ was regarded as one pseudo-diatom molecule; wave-packet propigation method has been employed to calculate the reaction probabilities; energy shifting,[28-30] methods are used to obtain approximate full dimensionality cumulative reaction probability for zero angular momentum $J$, and $J-K$ shifting methods [28-30] are employed to calculate total cumulative reaction probability for the full dimensionality rate constant;. Initial state selected reaction probabilities were calculated. We found that the excitation of the $\mathrm{H}_{2}$ substantially enhances the reactivity, whereas the excitation of the umbrella $x$ otion has the opposite effects. We also found that the $j_{1}=0$ initial rotational state of $H_{2}$ gives the largest reaction probability of the rotational excitation states of $\mathrm{H}_{2}$. On the otier hand, $j_{2}=1$ initial rotational state of $\mathrm{CH}_{3}$ has the largest reaction probability of the $\mathrm{CH}_{3}$ rotational states. Rate constant from our reduced dimensional calculation is larger than currently available experimental data, especially at the lower temperature range.

The quantum calculation mentioned above was done using the twelve degrees of freedom potential energy surface of Jordan and Gilbert [31]. This potential has a $C_{3 \nu}$ transition state with a collinear structure of $H-H-C$ and the center of mass of $H_{3}$. The vibrationally adiabatic barrier height of th is potential energy surface is $10.9 \mathrm{kcal} / \mathrm{mol}$ for the forward reaction, $\mathrm{H}+\mathrm{CH}_{4} \rightarrow \mathrm{H}_{2}+\mathrm{C}_{3}$ and $11.0 \mathrm{kcal} / \mathrm{mol}$ for the reverse reaction, $\mathrm{H}_{2}+\mathrm{CH}_{3} \rightarrow$ $\mathrm{H}+\mathrm{CH}_{4}$, respectively. This jotential energy surface is based in part on previous ab initio calculations of the saddle point[17], as well as on a previous, semiglobal potential used in variational transition state theory calculations of the rate constant[32]. The rate constant from the theoretical calculations of both the forward reaction[19, 20, 22-25] and the reverse 
reaction[27], comparing to the experiments, indicates that the barrier height on the JordanGilbert potential may be to s low.

In this paper, we extend the previous quantum dynamics study of the reaction $\mathrm{H}_{2}+\mathrm{CH}_{3} \rightarrow$ $\mathrm{H}+\mathrm{CH}_{4}[27]$ to include the isotopic reactions $\mathrm{HD}+\mathrm{CH}_{3}$ and $\mathrm{D}_{2}+\mathrm{CH}_{3}$, by treating them as diatom-diatom reaction s: stems and carry out full dimensional quantum dynamics calculations for those pseudo diat om-diatom reactions. Initial state selected reaction probability, cumulative reaction probability(CRP), and thermal rate constant are calculated. Ground state reaction probabilities, SRP, rate constants are compared in this report for these three reactions.

This paper is organized is follows: Section II briefly reviews the theory used in this calculation and the energy-s]ifting methods are also presented. Section III gives numerical results of the calculation, and section IV concludes.

\section{THEORY AND METHODS}

\section{A. Time-dependent wave-packet-propagation method}

This study employs the time-dependent wave-packet propagation method[27, 33-35] to carry out the quantum dynarics calculation. The two reactions, $\mathrm{HD}+\mathrm{CH}_{3}$ and $\mathrm{D}_{2}+\mathrm{CH}_{3}$ are treated as diatom-diatom reactions $H D\left(D_{2}\right)+C X$, as it is defined in the study of the reaction $\mathrm{H}_{2}+\mathrm{CH}_{3}[27]$, wher $\mathrm{X}$ is a pseudodiatom with the mass of the three nonreactive hydrogen atoms and located at their center of mass. The vibrational motion of CX approximately represents the umbrella motion of $\mathrm{CH}_{3}$, where the three hydrogen atoms move in phase, and the $\mathrm{C}-\mathrm{H}$ bonc length in $\mathrm{CH}_{3}$ is fixed at its equilibrium geometries. Thus the reduced dimensionality culculation for this pseudo diatom-diatom uses six degrees of freedom.

The mathematical treatment for these two reactions is the same as for the previous calculations of $\mathrm{H}_{2}+\mathrm{CH}_{3}$. A cirect product of a specific initial rotational-vibrational eigenfunctions of $H D\left(D_{2}\right)$ and $C X$ and a localized standard Gaussian function is chosen as the initial wave-packet. We emplcy the split-operator method to propagate the time-dependent wavefuntion. And the initial-state-selected reaction probability is extracted from the timedependent reactive flux in the standard way. 


\section{B. Cumulative reaction probability and thermal rate constant}

In order to obtain an ap proximate full dimensional (12D) cumulative reaction probability(CRP) for $J=0$ and thermal rate constant, we first calculate the reduced dimensional( $6 \mathrm{D}$ ) $\mathrm{CRP}(J=0)$, denoted $N^{J=0, r c}(E)$, by summing over all the initial-state-selected rovibrational reaction probability $P_{\nu_{1} \nu_{2} j_{1} j_{i}}^{J=0}(E)$,

$$
N^{\prime}=0, r d \dot{d}(E)=\sum_{\nu_{1}, \nu_{2}=0} \sum_{j_{1}, j_{2}=0} P_{\nu_{1} \nu_{2} j_{1} j_{2}}^{J=0}(E)
$$

where $E$ is the total Energy.

Our reduced dynamics calculation has six degrees of freedom, while the title reaction system has twelve degrees o: freedom. So the energy shifting method has to be applied to obtain the CRP for the full cimensionality using our reduced dimensional calculation. Thus

$$
N^{J=0, \text { full }}=\sum_{\nu_{1} \nu_{2}, \ldots, \nu_{6}} N^{J=0, r d}\left(E-E_{\nu_{1} \nu_{2}, \ldots, \nu_{6}}^{\ddagger}\right)
$$

here $\nu_{1} \nu_{2}, \ldots, \nu_{6}$ are the quantum numbers of the six vibrational degrees of freedom of the transition state, which are not included in our reduced dimensional calculation. These energies are given in the harmanic approximation by

$$
E_{\nu_{1} \nu_{2}, \ldots, \nu_{6}}^{\ddagger}=\sum_{i=1}^{6} \hbar \omega_{i}^{\ddagger}\left(\nu_{i}+\frac{1}{2}\right) .
$$

The J-K shifting method is employed here to calculate the CRP for nonzero total angular momentum $\mathrm{J}$. The total CRP, $N(E)$ is defined as the sum of CRP of all the open $\mathrm{J}$ and $\mathrm{K}$ channels

$$
N(E)=\sum_{J=0}(2 J+1) \sum_{K=-J}^{J} N^{J=0, f u l l}\left(E-E_{J K}^{\ddagger}\right),
$$

where $E_{J K}^{\ddagger}$ is the rigid rotor $\mathrm{r}$ (tational energy of the reaction system at the transition state. In the present case, the systen at transition state is a well defined symmetric top 


$$
E_{J K}^{\ddagger}=B^{\ddagger} J(J+1)+\left(A^{\ddagger}-B^{\ddagger}\right) K^{2} .
$$

$A^{\ddagger}$ and $B^{\ddagger}$ are the rotationa constants of $\mathrm{H}_{2} \mathrm{CH}_{3}$ at the full dimensional transition state.

The thermal rate constant can be computed exactly from the expression

$$
k(\tau)=\frac{1}{h Q_{\text {react }}} \int_{0}^{\infty} N(E) e^{-E / k_{B} T} d E .
$$

The above equation simplifies in the J-K shifting approximation, Eq. (4), to

$$
k(T)=\frac{Q_{\text {rot }}^{\ddagger}}{h Q_{\text {react }}} \int_{0}^{\infty} N(E)^{J=0, \text { full }} e^{-E / k_{B} T} d E,
$$

where $Q_{r o t}^{\ddagger}$ is the rotational f artition function of the reaction system at the transition state and $Q_{\text {react }}$ is the reactant partition function, which is written as a product of vibrational, rotational, and translational partition functions. As noted in Ref. [24], the consistent approach to calculate the vibrational partition function of $\mathrm{CH}_{3}$ is to combine the results of the reduced dimensional quantum calculations with the 'missing' vibrational frequencies which are not included in the reduced dimensionality. The purpose of doing this is to be consistent with the procedur that we use to convert the full dimensional CRP from the reduced dimensional CRP in Eq. (2).

\section{RESULTS AND DISC'USSION}

\section{A. Numerical aspects}

Sixty Sine basis functions cre used to expand the translational coordinates, 30 potential optimized vibrational functior.s for the $H D\left(D_{2}\right)$ vibrational coordinates, 8 potential optimized vibrational functions fo: the $C X$ vibrational coordinates and and total 1092 coupled angular momentum basis usec for the angular part. The rotational constants, $A^{\ddagger}$ and $B^{\ddagger}$ in Eq. (5), are $5.12 \mathrm{~cm}^{-1}$ and $1.33 \mathrm{~cm}^{-1}$ for $H D+C H_{3}$ system; $5.12 \mathrm{~cm}^{-1}$ and $1.23 \mathrm{~cm}^{-1}$ for $\mathrm{D}_{2}+\mathrm{CH}_{3}$ system. The ribrational frequencies that are 'missing' in our reduced dimensional calculations at the transition state are: $589 \mathrm{~cm}^{-1}(2), 1439 \mathrm{~cm}^{-1}(2), 2959 \mathrm{~cm}^{-1}$ and $3092 \mathrm{~cm}^{-1}(2)[24]$. These missing' vibrational frequencies are used to obtain the full dimensionality CRP for J=0 ir Eq. (2). 


\section{B. Initial-state-select $€ d$ probability}

Fig. 1 shows the reaction probabilities for the frst four initial vibrational states of $H D\left(\nu_{1}\right.$, $j_{1}=0$ ) with $C X$ at ground sté te as a function of kinetic energy. As seen, vibrational excitation of $H D$ enhances this reactirity with $\nu_{1}=3$ having the largest reaction probability. And in addition, vibrational excitation of $H D$ also enhances the reactivity by lowing the reaction threshold. The same trend of reaction probabilities is also shown in Fig. 2 for reaction $D_{2}\left(\nu_{1}, j_{1}=0\right)+C X\left(\nu_{2}=0, j=0\right)$.

The reaction probabilities of vibrational excitation of $C X\left(\nu_{2}, j_{2}=0, \nu_{1}=j_{1}=0\right)$ are plotted in Fig.3 and Fig.4 respectively for $\mathrm{HD}+\mathrm{CH} 3$ and $\mathrm{D}_{2}+\mathrm{CH}_{3}$. Comparing to Fig.1, These figures simply tell us that excitations of the umbrella motion of $C X$ hinder the reactivity due to $C X$ mainly functions as a spector in this reaction. The more of the $\mathrm{CH}_{3}$ vibrates, the harder for atoms in $H D$ and $D_{2}$ to 'catch' the $\mathrm{C}$ atom in $\mathrm{CH}_{3}$.

The comparison of reaction probabilities for the three reactions, $\mathrm{H}_{2}\left(\mathrm{HD}, \mathrm{D}_{2}\right)+\mathrm{CH}_{3}$ with all reactants at their ground ro-vibrational states are plotted in Fig. 5. The isotopic effect has been shown in this figure that the $\mathrm{H}_{2}+\mathrm{CH}_{3}$ reaction has the smallest threshold, and $\mathrm{D}_{2}+\mathrm{CH}_{3}$ has the highest. Ine can say, the $\mathrm{H}_{2}+\mathrm{CH}_{3}$ reaction has the lowest adiabatic barrier because of the largest zero-point energy and $\mathrm{D}_{2}+\mathrm{CH}_{3}$ reaction has higest adiabatic barrier. So at relatively lowe: kinetic energies, $\mathrm{H}_{2}+\mathrm{CH}_{3}$ reaction has the largest reaction probability, $\mathrm{D}_{2}+\mathrm{CH}_{3}$ reacticn has the smallest reaction probability. However, the reaction probablities have the reverse order at relatively high kinetic energies. This is also caused by the isotopic effect because $D_{:}$is the heaviest among the three hydorgen isotopes. And at relatively high kinetic energie;, it moves slower than the other two, $H_{2}$ and $H D$, respect to $\mathrm{CH}_{3}$, so it's easiler to get conbined with the $\mathrm{C}$ atom in $\mathrm{CH}_{3}$ to let reaction occur.

\section{Cumulative reaction probability and thermal rate constant}

A normal mode analysis is performed to determine the 'missing' modes in our reduced dimensional quantum calculation, as also seen in Ref.[24]. Then Eq.(2) is used to obtain the full dimensional CRP for $:=0$. For total scattering energy up to $2.0 \mathrm{eV}$, all the missing modes given in Section A. contribute to convert the full dimensional CRP from the sixdegree-of-freedom quantum calculation. And the comparison of full dimensional converted 
$\mathrm{CPR}(\mathrm{J}=0)$ is shown in Fig. 6 as a function of the total energy, which includes the energy difference $0.702 \mathrm{eV}$ which rot included in the $6 \mathrm{D}$ reduced dimensional ZPE. Using the harmonic approximation of the 'missing' degrees of freedom in $\mathrm{CH}_{3}$ is consistent with using this approximation in the energy-shifting expression in Eq. (2). This figure shows that $D_{2}+C_{3}$ has the largest $\mathrm{C} Z \mathrm{P}(\mathrm{J}=0)$, which is expected since $\mathrm{D}_{2}+\mathrm{CH}_{3}$ reaction has more open channels (initial rotational-vibrational states) than the other two in the energy rangy we are interested here.

Our previous study[27] sh.owed the rate constant of $\mathrm{H}_{2}+\mathrm{CH}_{3}$ is larger the experiment rates, especially in the low semperature, this indicates the barrier on the Jordan-Gilbert potential is too low. Comparision of our present rate constants of the three reactions shows $\mathrm{H}_{2}+\mathrm{CH}_{3}$ the largest rate cc nstant, then $\mathrm{HD}+\mathrm{CH}_{3}$ and $\mathrm{D}_{2}+\mathrm{CH}_{3}$ the smallest in Fig. 7 . In other words, the isotopic effect shows, from these three reactions, that the larger of the mass of the reactant particle the smaller reactivity.

\section{SUMMARY AND CONCLUSIONS}

In this study, we perform the isotopic chemical reaction study of $\mathrm{HD}+\mathrm{CH}_{3}$ and $\mathrm{D}_{2}+$ $\mathrm{CH}_{3}$, which is an extension study of the $\mathrm{H}_{2}+\mathrm{CH}_{3}$ reaction. Time-dependent wave-packet propagation method is carried out in six-degree-of freedom for the title reactions. The energy-shifting method has be em employed to obtain the full dimensional cumulative reaction probability for $\mathrm{J}=0$. And $\mathrm{J}-\mathrm{K}$ shifting approach is applied to this CRP to obtain the thermal rate constant which is compred with different experiments. A potential surface due to Jordan and Gilbert, which has been used in previous quantum calculation of the forward reaction $\mathrm{H}+\mathrm{CH}_{4} \rightarrow \mathrm{H}_{2}+\mathrm{CH}_{3}$, was used in these calculations.

Initial-state-selected reaction probability and the cumulative reaction probability were calculated for zero total angular momentum. The vibrational excitations of $H D\left(D_{2}\right)$ substantially enhance the reactio a probabilities. On the contrary, the vibrational excitation of $\mathrm{CH}_{3}$ causes the opposite effec's in these reactions. The $\mathrm{H}_{2}+\mathrm{CH}_{3}$ reaction has the smallest adiabatic barrier due to the largest ZPE because of the isotopic effect. This study also shows at relatively high kinetic eners,,$D_{2}+C H_{3}$ is the most reactive one.

An energy shifting method was used to convert the reduced dimensional CRP for zero total angular momentum to a full dimensional one. Our rate constant comparison shows 
$\mathrm{H}_{2}+\mathrm{CH}_{3}$ reaction has the largest rate constant against the smallest of $\mathrm{D}_{2}+\mathrm{CH}_{3}$.

\section{Acknowledgments}

This work is supported by NASA prime contract NAS2-00062. The author would like to thank Dr. Winifred Huo anc. Dr. James Stallcop for very helpful discussions on the subject. 
[1] J. Warnatz, in Combustiin Chemistry, edited by W.C. Jr. (Springer-Verlag, New York, 1984).

[2] C.P. Fenimore and G.W. Jones J. Phys. Chem. 65, 200 (1961).

[3] J. Peeters, G. Mahnen, 14th Symposium (international) on Combustion, (The Combustion Institute, Pittsburgh, 19;3).

[4] J.C. Biordi, C.P. Lazzara and J.F. Papp, Combust. Flame 26, 57 (1976).

[5] P. Roth and T. Just, Ber Bunsenges. Phys. Chem. 79, 682 (1975).

[6] W. Moller and E. Mozzhushin and H.G. Wagner, Ber. Bunsenges. Phys. Chem. 90, 854 (1980).

[7] H.J. Baeck, K.S. Shin, H. Yang, Z. Qing, V. Lissianski and W.C Gardiner, Jr., J. Phys. Chem. 99, 15925 (1995).

[8] J.W. Sutherland M.C. Su and J.V. Micheal, Inter. J. Chem. Kinet. 33, 69 (2001).

[9] M.J. Rabinowitz, J.W. Sutherland, P.M. Patterson and R.B. Klemm, J. Phys. Chem. 95, 674 (1991).

[10] V.D. Knyazev, A. Bencsua, S.I. Stoliarov and I.R. Slagle J. Phys. Chem. 100, 11346 (1996).

[11] R. Shaw, J. Phys. Chem. Ref. Data 7, 1179 (1978).

[12] A. Fahr, P.S. Monks, L.J. Stief and A.H. Laufer, Icarus 116,, 415 (1995).

[13] T.C. Clark, J.E. Dove, J. Them. Phys. 51, 2147 (1973).

[14] L.M. Raff J. Chem. Phys. 60, 2220 (1974).

[15] G.C. Schatz, A.F. Wagner and T.H. Dunning J. Phys. Chem. 88, 221 (1984).

[16] R. Steckler, K.J. Dykema, K.J. Brown, F.B. Hancock and D.G. Truhlar J. Chem. Phys. 87, 7024 (1987).

[17] T. Joseph, R. Steckler and D.G. Truhlar J. Chem. Phys. 87, 1036 (1987).

[18] J. Pu and D.G. Truhlar, J. Chem. Phys. 116, 1468 (2002).

[19] T. Takayanagi, J. Chem. Phys. 104, 2237 (1996).

[20] H.-G. Yu and G. Nyman, J. Chem. Phys. 111, 3508 (1999).

[21] M.L. Wang, Y. Li, J.Z.H. Zhang and D.H. Zhang, J. Chem. Phys. 113, 1802 (2000).

[22] F. Huarte-Larrañaga and I: Manthe J. Chem. Phys. 113, 5115 (2000)

[23] J.M. Bowman, D.Y. Wang, X.C. Huang, F. Huarte-Larrañaga and U. Manthe J. Chem. Phys. 114, 9683 (2001).

[24] D.Y. Wang and J.M. Bowr an J. Chem. Phys. 115, 2055 (2001). 
[25] F. Huarte-Larrañaga and U. Manthe J. Chem. Phys. 116, 2863 (2002)

[26] M.L. Wang and J.Z.H. Zhang J. Chem. Phys. 116, 6497 (2002).

[27] D.Y. Wang, submitted to J. Chem. Phys. .

[28] J.M. Bowman, J. Phys. Chem. 95, 4950 (1991).

[29] J.M. Bowman, in Reaction and Molecular Dynamics Proceedings of the European School on Computational Chemistr., Perugia, Italy, July(1999), edited by A. Lagana and A. Riganelli (Springer, New York, 20C0).

[30] J.M. Bowman and A. F. Wagner, in The Theory of Chemical Reaction Dynamics, edited by D.C. Clary (Reidel, Dord:echt, 1986).

[31] M. Jordan and R. Gilber1, J. Chem. Phys. 102, 5669 (1995).

[32] G.C. Schatz, S.P. Walch ind A.F. Wagner, J. Chem. Phys. 73, 4536 (1980).

[33] D.H. Zhang and J.Z.H. Zliang, J. Chem. Phys. 100, 2687 (1993).

[34] D.H. Zhang and J.Z.H. Zhang, J. Chem. Phys. 101, 1446 (1994).

[35] Y.C. Zhang, D. Zhang, W. Li, Q.G. Zhang, D.Y. Wang, D.H. Zhang and J.Z.H. Zhang J. Phys. Chem. 99, 16824 (1995). 
Fig. 1 The initial state selscted reaction probability for the reaction $H D\left(\nu_{1}, j_{1}=0\right)+$ $\mathrm{CH}_{3}\left(\nu_{2}=j_{2}=0\right)$ as a function of kinetic energy $(\mathrm{J}=0)$.

Fig. 2 Same as Fig. 1 but fo: the reaction $D_{2}\left(\nu_{1}, j_{1}=0\right)+C H_{3}\left(\nu_{2}=j_{2}=0\right)$.

Fig. 3 The initial state sele ted reaction probability for the reaction $H D\left(\nu_{1}=0, j_{1}=0\right)+$ $\mathrm{CH}_{3}\left(\nu_{1}, j_{2}=0\right)$ as a fur ction of kinetic energy $(\mathrm{J}=0)$.

Fig. 4 Same as Fig. 3 but for the reaction $D_{2}\left(\nu_{1}=0, j_{1}=0\right)+C H_{3}\left(\nu_{1}, j_{2}=0\right)$.

Fig. 5 Comparison of the ground state reaction probability for all three isotopic reaction: $\mathrm{H}_{2}\left(\mathrm{HD}, \mathrm{D}_{2}\right)+\mathrm{CH}_{3}$ reaction as a function of kinetic energy.

Fig. 6 Comparison of cumulctive reaction probabilities $N^{J=0, f u l l}(E)$ for all three isotopic reactions as a function of total energy.

Fig. 7 Comparison of all three isotopic thermal rate constant. 


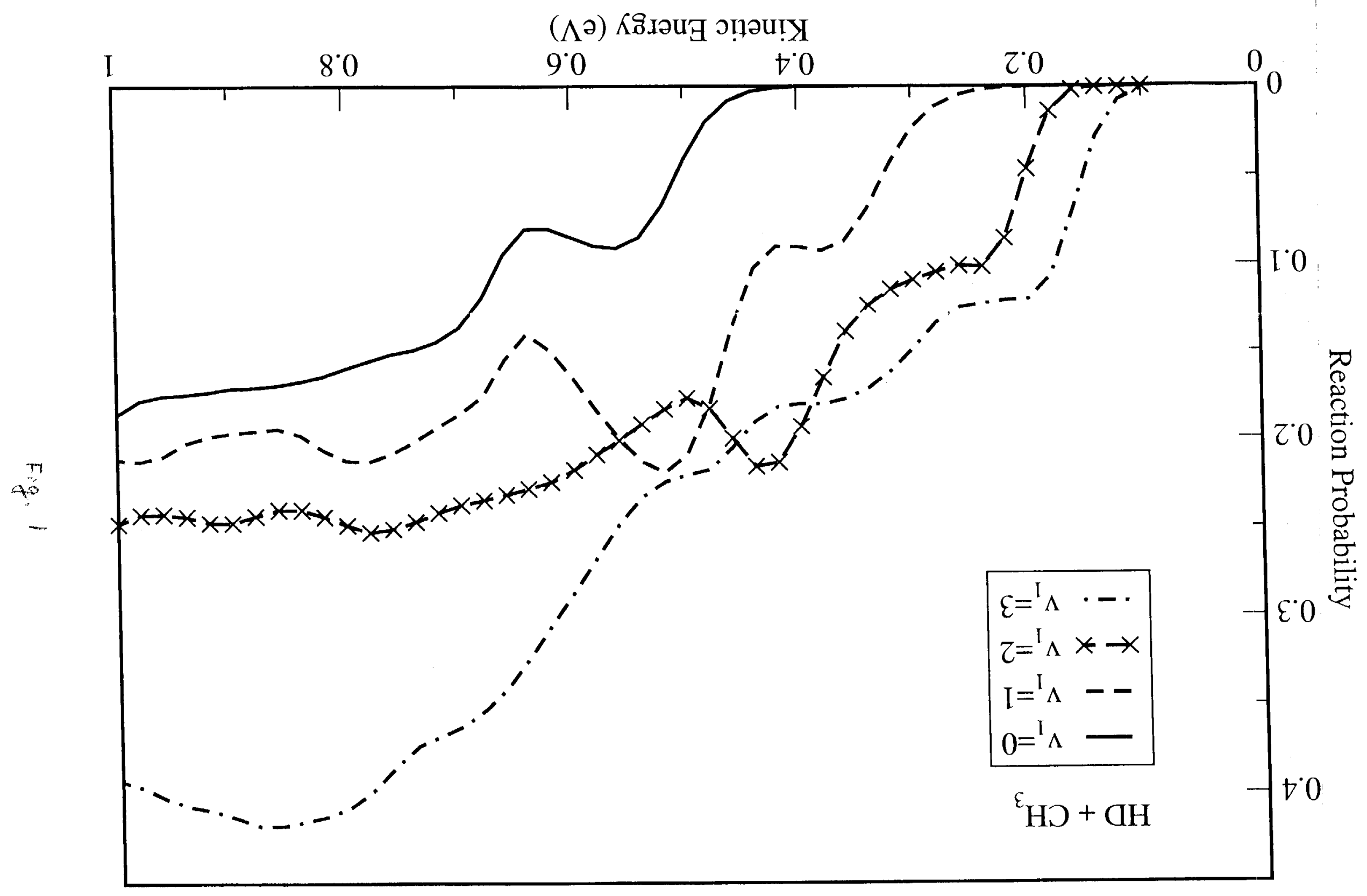




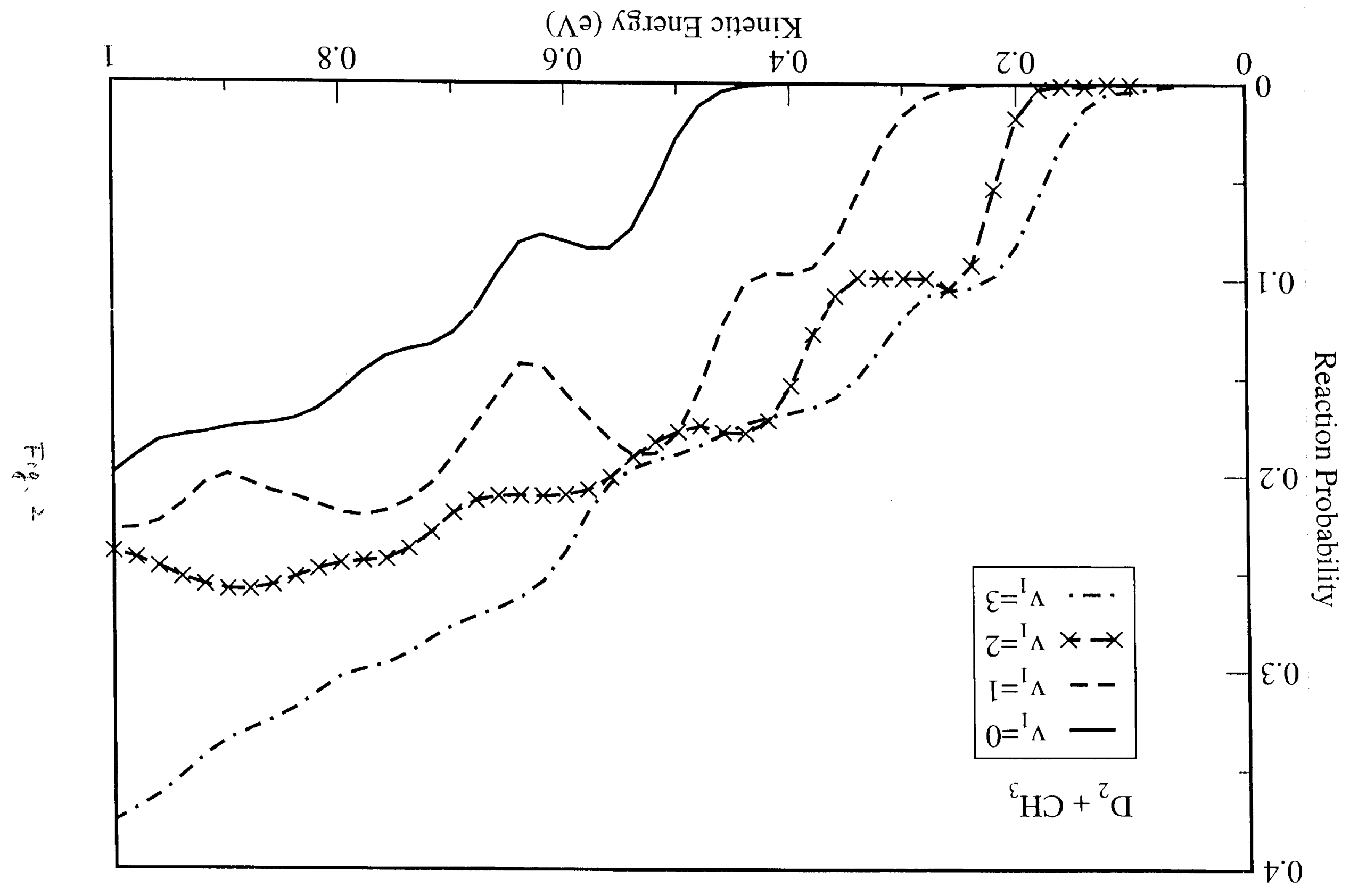




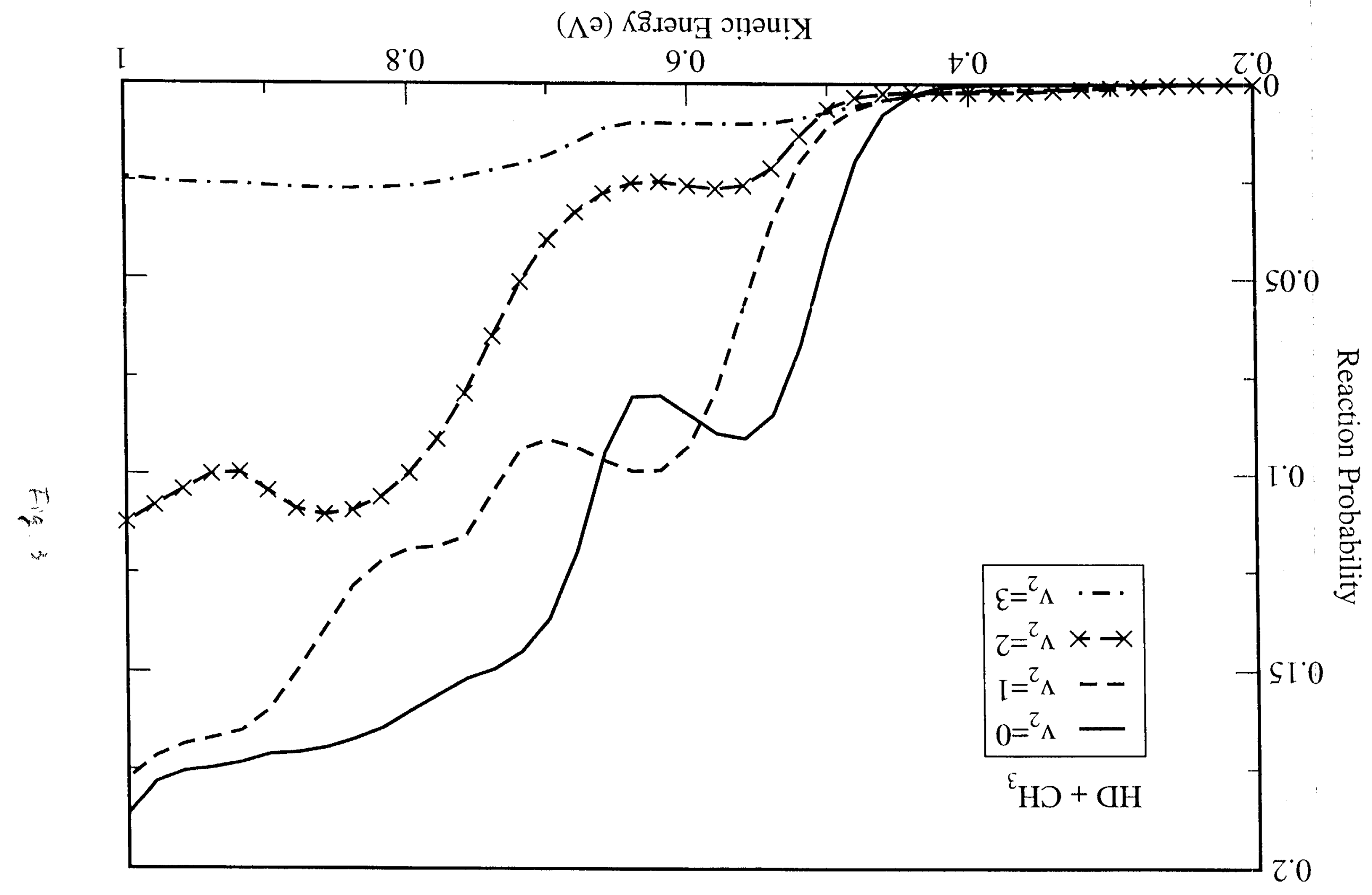




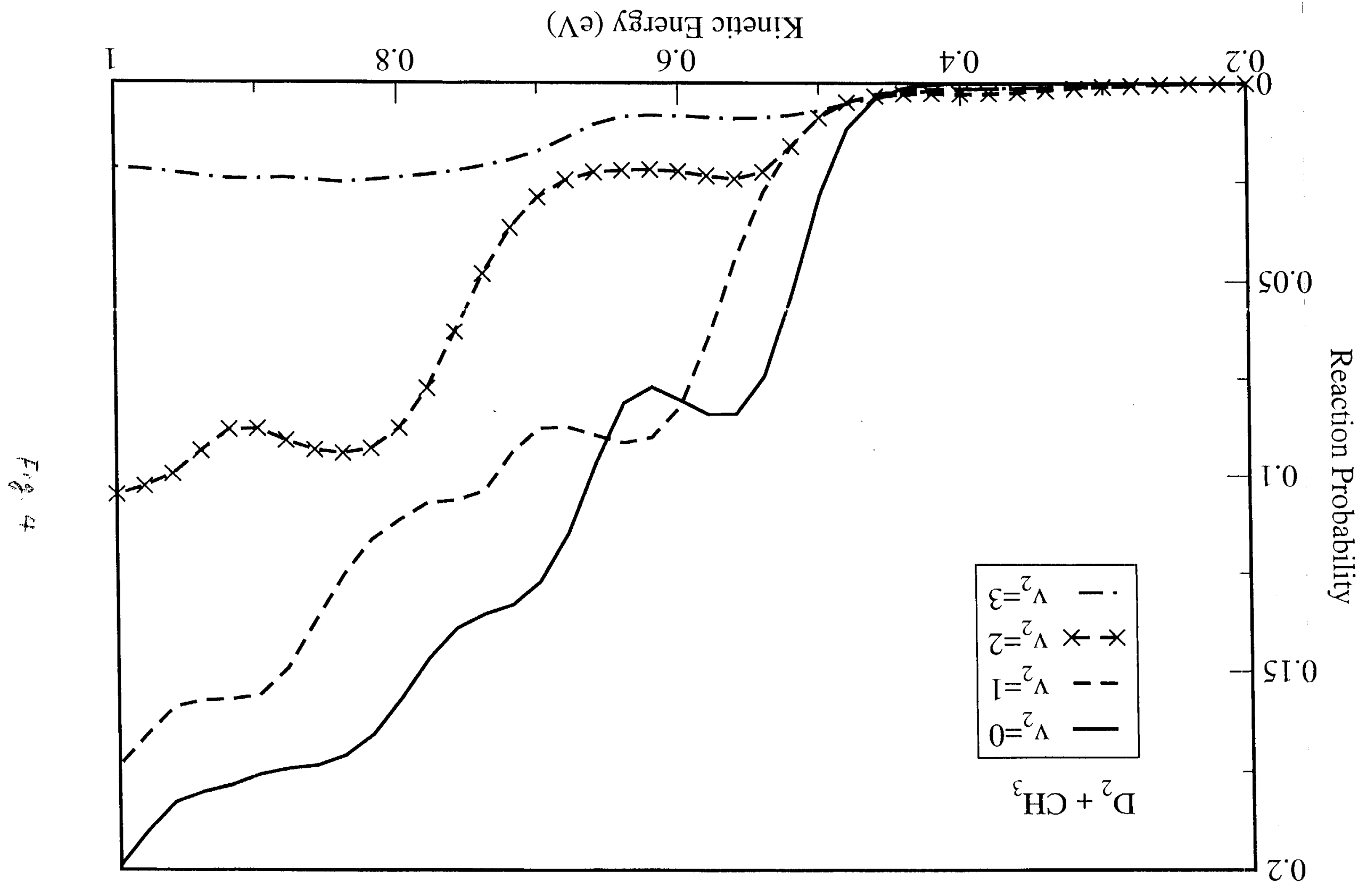




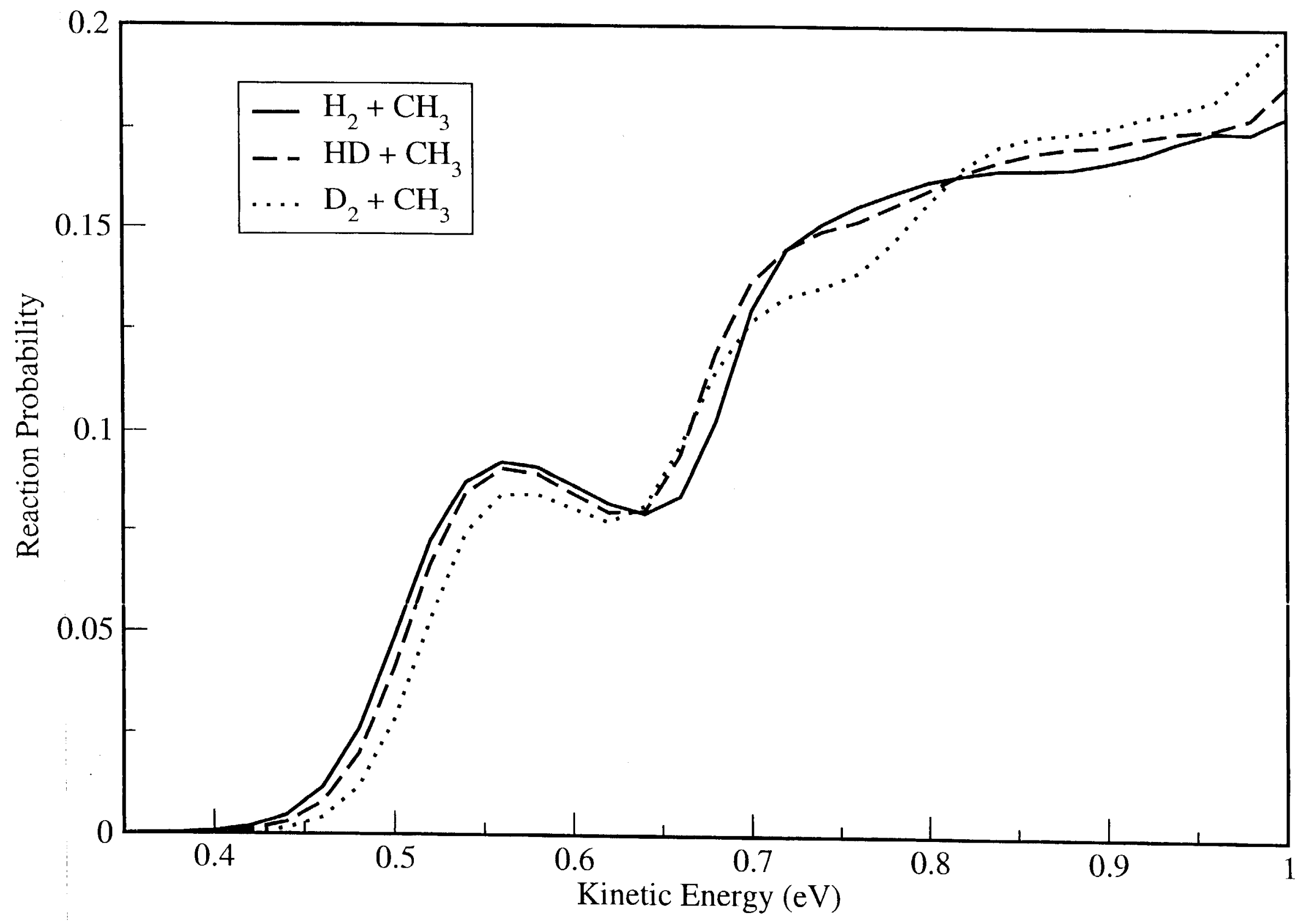

4 


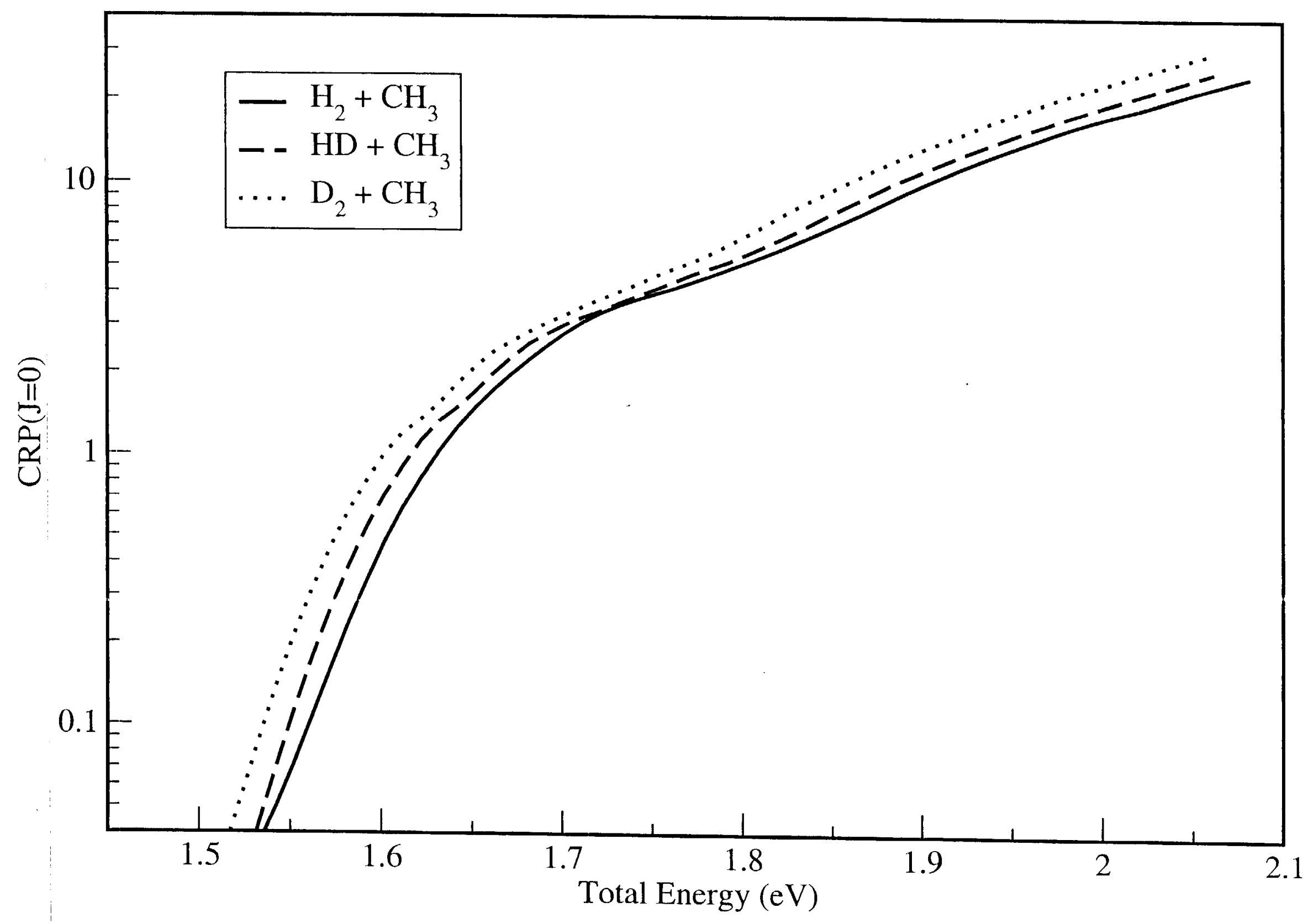




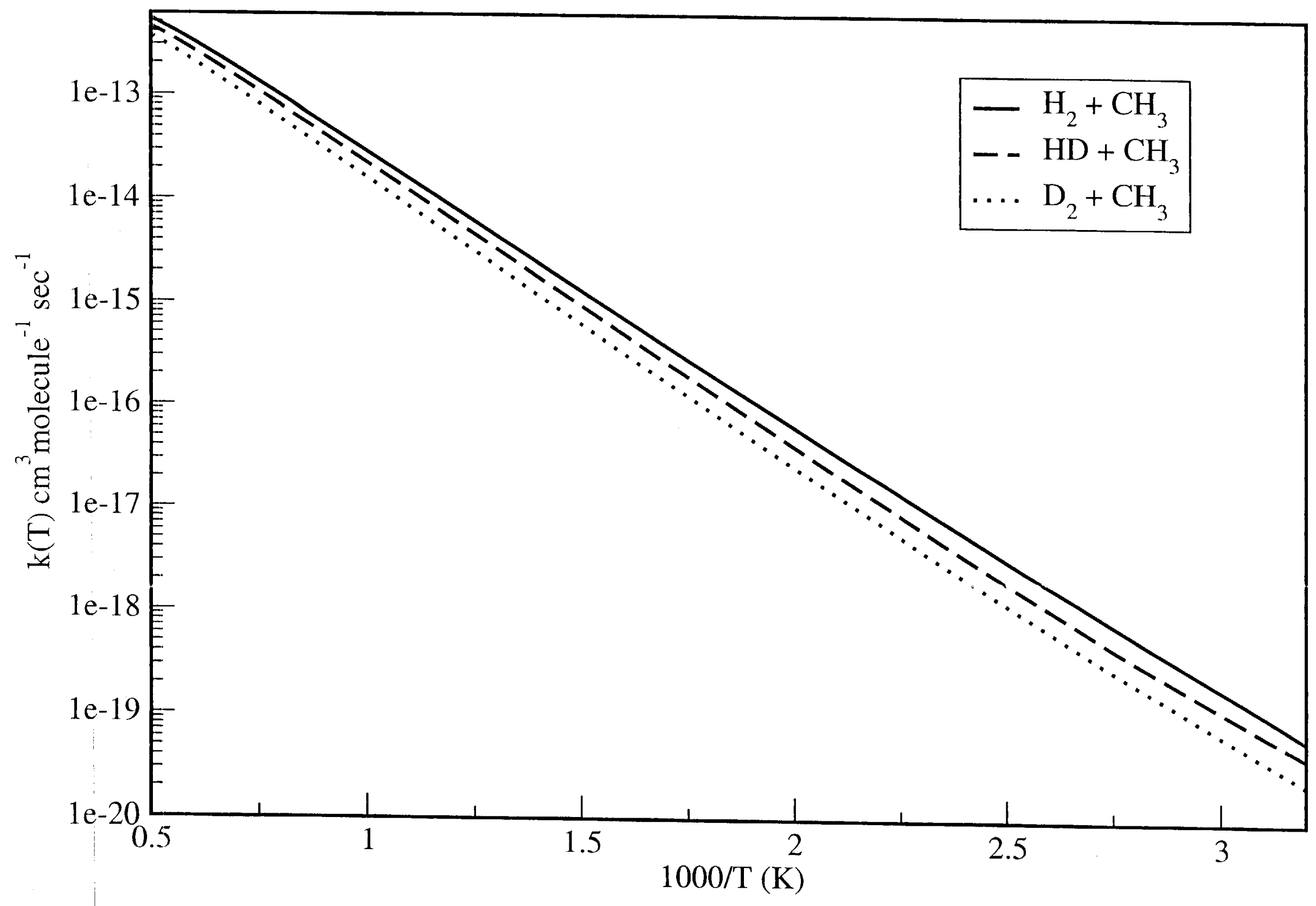

